S. S. Dragomir*

\title{
Some Hermite-Hadamard type inequalities for operator convex functions and positive maps
}

https://doi.org/10.1515/spma-2019-0005

Received February 10, 2019; accepted March 27, 2019

Abstract: In this paper we establish some inequalities of Hermite-Hadamard type for operator convex functions and positive maps. Applications for power function and logarithm are also provided.

Keywords: Jensen's inequality,Hermite-Hadamard inequality, Positive maps, Operator convex functions, Arithmetic mean-Geometric mean inequality

MSC: 47A63, 47A30, 15A60

\section{Introduction}

A real valued continuous function $f$ on an interval $I$ is said to be operator convex (operator concave) if

$$
f((1-\lambda) A+\lambda B) \leq(\geq)(1-\lambda) f(A)+\lambda f(B)
$$

in the operator order, for all $\lambda \in[0,1]$ and for every selfadjoint operator $A$ and $B$ on a Hilbert space $H$ whose spectra are contained in $I$. Notice that a function $f$ is operator concave if $-f$ is operator convex.

A real valued continuous function $f$ on an interval $I$ is said to be operator monotone if it is monotone with respect to the operator order, i.e., $A \leq B$ with $\operatorname{Sp}(A), \operatorname{Sp}(B) \subset I$ imply $f(A) \leq f(B)$.

For some fundamental results on operator convex (operator concave) and operator monotone functions, see for instance [12] and the references therein.

As examples of such functions, we note that $f(t)=t^{r}$ is operator monotone on $[0, \infty)$ if and only if $0 \leq r \leq$ 1. The function $f(t)=t^{r}$ is operator convex on $(0, \infty)$ if either $1 \leq r \leq 2$ or $-1 \leq r \leq 0$ and is operator concave on $(0, \infty)$ if $0 \leq r \leq 1$. The logarithmic function $f(t)=\ln t$ is operator monotone and operator concave on $(0, \infty)$. The entropy function $f(t)=-t \ln t$ is operator concave on $(0, \infty)$. The exponential function $f(t)=e^{t}$ is neither operator convex nor operator monotone.

In [4], see also [5, p. 60], we established the following Hermite-Hadamard type inequality for operator convex functions:

\footnotetext{
^Corresponding Author: S. S. Dragomir: Mathematics, College of Engineering \& Science, Victoria University, PO Box 14428 , Melbourne City, MC 8001, Australia, E-mail: sever.dragomir@vu.edu.au and School of Computer Science \& Applied Mathematics, University of the Witwatersrand, Private Bag 3, Johannesburg 2050, South Africa

http://rgmia.org/dragomir
} 
Theorem 1. Let $f: I \rightarrow \mathbb{R}$ be an operator convex function on the interval $I$. Then for any selfadjoint operators $A$ and $B$ with spectra in $I$ we have the inequality

$$
\begin{aligned}
f\left(\frac{A+B}{2}\right) & \leq \frac{1}{2}\left[f\left(\frac{3 A+B}{4}\right)+f\left(\frac{A+3 B}{4}\right)\right] \\
& \leq \int_{0}^{1} f((1-t) A+t B) d t \\
& \leq \frac{1}{2}\left[f\left(\frac{A+B}{2}\right)+\frac{f(A)+f(B)}{2}\right] \leq \frac{f(A)+f(B)}{2} .
\end{aligned}
$$

For recent related results on operator Hermite-Hadamard type inequalities, see [1]-[2], [5]-[10] and [13].

Let $H$ be a complex Hilbert space and $\mathcal{B}(H)$, the Banach algebra of bounded linear operators acting on $H$. We denote by $\mathcal{B}^{+}(H)$ the convex cone of all positive operators on $H$ and by $\mathcal{B}^{++}(H)$ the convex cone of all positive definite operators on $H$.

Let $H, K$ be complex Hilbert spaces. Following [3] (see also [12, p. 18]) we can introduce the following definition:

Definition 1. A map $\Phi: \mathcal{B}(H) \rightarrow \mathcal{B}(K)$ is linear if it is additive and homogeneous, namely

$$
\Phi(\lambda A+\mu B)=\lambda \Phi(A)+\mu \Phi(B)
$$

for any $\lambda, \mu \in \mathbb{C}$ and $A, B \in \mathcal{B}(H)$. The linear map $\Phi: \mathcal{B}(H) \rightarrow \mathcal{B}(K)$ is positive if it preserves the operator order, i.e. if $A \in \mathcal{B}^{+}(H)$ then $\Phi(A) \in \mathcal{B}^{+}(K)$. We write $\Phi \in \mathfrak{P}[\mathcal{B}(H), \mathcal{B}(K)]$. The linear map $\Phi: \mathcal{B}(H) \rightarrow \mathcal{B}(K)$ is normalised if it preserves the identity operator, i.e. $\Phi\left(1_{H}\right)=1_{K}$. We write $\Phi \in \mathfrak{P}_{N}[\mathcal{B}(H), \mathcal{B}(K)]$.

We observe that a positive linear map $\Phi$ preserves the order relation, namely

$$
A \leq B \text { implies } \Phi(A) \leq \Phi(B)
$$

and preserves the adjoint operation $\Phi\left(A^{\star}\right)=\Phi(A)^{\star}$. If $\Phi \in \mathfrak{P}_{N}[\mathcal{B}(H), \mathcal{B}(K)]$ and $\alpha 1_{H} \leq A \leq \beta 1_{H}$, then $\alpha 1_{K} \leq \Phi(A) \leq \beta 1_{K}$.

If the map $\Psi: \mathcal{B}(H) \rightarrow \mathcal{B}(K)$ is linear, positive and $\Psi\left(1_{H}\right) \in \mathcal{B}^{++}(K)$ then by putting $\Phi=$ $\Psi^{-1 / 2}\left(1_{H}\right) \Psi \Psi^{-1 / 2}\left(1_{H}\right)$ we get that $\Phi \in \mathfrak{P}_{N}[\mathcal{B}(H), \mathcal{B}(K)]$, namely it is also normalised.

The following Jensen's type result is well known [3]:

Theorem 2 (Davis-Choi-Jensen's Inequality). Let $f: I \rightarrow \mathbb{R}$ be an operator convex function on the interval I and $\Phi \in \mathfrak{P}_{N}[\mathcal{B}(H), \mathcal{B}(K)]$, then for any selfadjoint operator $A$ whose spectrum is contained in $I$ we have

$$
f(\Phi(A)) \leq \Phi(f(A)) .
$$

We observe that if $\Psi \in \mathfrak{P}[\mathcal{B}(H), \mathcal{B}(K)]$ with $\Psi\left(1_{H}\right) \in \mathcal{B}^{++}(K)$, then by taking $\Phi=\Psi^{-1 / 2}\left(1_{H}\right) \Psi \Psi^{-1 / 2}\left(1_{H}\right)$ in (1.2) we get

$$
f\left(\Psi^{-1 / 2}\left(1_{H}\right) \Psi(A) \Psi^{-1 / 2}\left(1_{H}\right)\right) \leq \Psi^{-1 / 2}\left(1_{H}\right) \Psi(f(A)) \Psi^{-1 / 2}\left(1_{H}\right) .
$$

If we multiply both sides of this inequality by $\Psi^{1 / 2}\left(1_{H}\right)$ we get the following Davis-Choi-Jensen's inequality for general positive linear maps:

$$
\Psi^{1 / 2}\left(1_{H}\right) f\left(\Psi^{-1 / 2}\left(1_{H}\right) \Psi(A) \Psi^{-1 / 2}\left(1_{H}\right)\right) \Psi^{1 / 2}\left(1_{H}\right) \leq \Psi(f(A)) .
$$

In this paper, motivated by the above results, we establish some inequalities of Hermite-Hadamard type for operator convex functions and positive maps. Applications for power function and logarithm are also provided. 


\section{Refinements of HH-Inequality}

Let $f: I \rightarrow \mathbb{R}$ be an operator convex function on the interval $I$ and two selfadjoint operators $A$ and $B$ with spectra in $I$ and $\Phi \in \mathfrak{P}_{N}[\mathcal{B}(H), \mathcal{B}(K)]$. We know that $\Phi$ is continuous, see for instance [11, Proposition 2.8]. By taking the positive map $\Phi$ in (1.1) and using the continuity property of $\Phi$, we have

$$
\begin{aligned}
\Phi\left(f\left(\frac{A+B}{2}\right)\right) & \leq \frac{1}{2}\left[\Phi\left(f\left(\frac{3 A+B}{4}\right)\right)+\Phi\left(f\left(\frac{A+3 B}{4}\right)\right)\right] \\
& \leq \int_{0}^{1} \Phi(f((1-t) A+t B)) d t \\
& \leq \frac{1}{2}\left[\Phi\left(f\left(\frac{A+B}{2}\right)\right)+\frac{\Phi(f(A))+\Phi(f(B))}{2}\right] \\
& \leq \frac{\Phi(f(A))+\Phi(f(B))}{2} .
\end{aligned}
$$

If we write the inequality (2.1) for $\Phi(A)$ and $\Phi(B)$ then we also have

$$
\begin{aligned}
f\left(\frac{\Phi(A)+\Phi(B)}{2}\right) & \leq \frac{1}{2}\left[f\left(\frac{3 \Phi(A)+\Phi(B)}{4}\right)+f\left(\frac{\Phi(A)+3 \Phi(B)}{4}\right)\right] \\
& \leq \int_{0}^{1} f((1-t) \Phi(A)+t \Phi(B)) d t \\
& \leq \frac{1}{2}\left[f\left(\frac{\Phi(A)+\Phi(B)}{2}\right)+\frac{f(\Phi(A))+f(\Phi(B))}{2}\right] \\
& \leq \frac{f(\Phi(A))+f(\Phi(B))}{2} .
\end{aligned}
$$

It is then natural to ask how the following integrals

$$
\int_{0}^{1} \Phi(f((1-t) A+t B)) d t \text { and } \int_{0}^{1} f((1-t) \Phi(A)+t \Phi(B)) d t
$$

do compare?

The following simple result holds:

Theorem 3. Let $f: I \rightarrow \mathbb{R}$ be an operator convex function on the interval $I$. Then for any selfadjoint operators $A$ and $B$ with spectra in $I$ and $\Phi \in \mathfrak{P}_{N}[\mathcal{B}(H), \mathcal{B}(K)]$ we have

$$
\int_{0}^{1} f((1-t) \Phi(A)+t \Phi(B)) d t \leq \int_{0}^{1} \Phi(f((1-t) A+t B)) d t .
$$

Proof. By (1.2) we have

$$
f((1-t) \Phi(A)+t \Phi(B))=f(\Phi(((1-t) A+t B))) \leq \Phi(f((1-t) A+t B))
$$

for any $t \in[0,1]$.

By integrating this inequality on $[0,1]$ and using the continuity property of $\Phi$ we get the desired result (2.3).

We define by $\mathfrak{P}_{I}[\mathcal{B}(H), \mathcal{B}(K)]$ the convex cone of all linear, positive maps $\Psi$ with $\Psi\left(1_{H}\right) \in \mathcal{B}^{++}(K)$, namely $\Psi\left(1_{H}\right)$ is positive invertible operator in $K$. 
Corollary 1. Let $f: I \rightarrow \mathbb{R}$ be an operator convex function on the interval I and selfadjoint operators $A$ and $B$ with spectra in I. If $\Psi \in \mathfrak{P}_{I}[\mathcal{B}(H), \mathcal{B}(K)]$, then we have

$$
\begin{gathered}
\Psi^{1 / 2}\left(1_{H}\right)\left(\int_{0}^{1} f\left(\Psi^{-1 / 2}\left(1_{H}\right)((1-t) \Psi(A)+t \Psi(B)) \Psi^{-1 / 2}\left(1_{H}\right)\right) d t\right) \Psi^{1 / 2}\left(1_{H}\right) \\
\leq \int_{0}^{1} \Psi(f((1-t) A+t B)) d t .
\end{gathered}
$$

Proof. If we write the inequality (2.3) for $\Phi=\Psi^{-1 / 2}\left(1_{H}\right) \Psi \Psi^{-1 / 2}\left(1_{H}\right)$, then we get

$$
\begin{aligned}
& \int_{0}^{1} f\left((1-t) \Psi^{-1 / 2}\left(1_{H}\right) \Psi(A) \Psi^{-1 / 2}\left(1_{H}\right)+t \Psi^{-1 / 2}\left(1_{H}\right) \Psi(B) \Psi^{-1 / 2}\left(1_{H}\right)\right) d t \\
& \leq \int_{0}^{1} \Psi^{-1 / 2}\left(1_{H}\right) \Psi(f((1-t) A+t B)) \Psi^{-1 / 2}\left(1_{H}\right) d t,
\end{aligned}
$$

that can be written as

$$
\begin{aligned}
& \int_{0}^{1} f\left(\Psi^{-1 / 2}\left(1_{H}\right)((1-t) \Psi(A)+t \Psi(B)) \Psi^{-1 / 2}\left(1_{H}\right)\right) d t \\
& \leq \Psi^{-1 / 2}\left(1_{H}\right)\left(\int_{0}^{1} \Psi(f((1-t) A+t B)) d t\right) \Psi^{-1 / 2}\left(1_{H}\right) .
\end{aligned}
$$

Finally, if we multiply both sides of this inequality by $\Psi^{1 / 2}\left(1_{H}\right)$, then we get the desired result (2.4).

The following representation result holds.

Lemma 1. Let $f: I \rightarrow \mathbb{C}$ be a continuous function on the interval $I$ and two selfadjoint operators $A$ and $B$ with spectra in $I$. Then for any $\lambda \in[0,1]$ we have the representation

$$
\begin{aligned}
\int_{0}^{1} f((1-t) A+t B) d t & =(1-\lambda) \int_{0}^{1} f[(1-t)((1-\lambda) A+\lambda B)+t B] d t \\
& +\lambda \int_{0}^{1} f[(1-t) A+t((1-\lambda) A+\lambda B)] d t .
\end{aligned}
$$

Proof. For $\lambda=0$ and $\lambda=1$ the equality (2.5) is obvious.

Let $\lambda \in(0,1)$. Observe that

$$
\int_{0}^{1} f[(1-t)(\lambda B+(1-\lambda) A)+t B] d t=\int_{0}^{1} f[((1-t) \lambda+t) B+(1-t)(1-\lambda) A] d t
$$

and

$$
\int_{0}^{1} f[t(\lambda B+(1-\lambda) A)+(1-t) A] d t=\int_{0}^{1} f[t \lambda B+(1-\lambda t) A] d t .
$$

If we make the change of variable $u:=(1-t) \lambda+t$ then we have $1-u=(1-t)(1-\lambda)$ and $d u=(1-\lambda) d u$. Then

$$
\int_{0}^{1} f[((1-t) \lambda+t) B+(1-t)(1-\lambda) A] d t=\frac{1}{1-\lambda} \int_{\lambda}^{1} f[u B+(1-u) A] d u .
$$


If we make the change of variable $u:=\lambda t$ then we have $d u=\lambda d t$ and

$$
\int_{0}^{1} f[t \lambda B+(1-\lambda t) A] d t=\frac{1}{\lambda} \int_{0}^{\lambda} f[u B+(1-u) A] d u .
$$

Therefore

$$
\begin{aligned}
(1-\lambda) \int_{0}^{1} f[(1-t)(\lambda B+(1-\lambda) A)+t B] d t+ & \lambda \int_{0}^{1} f[t(\lambda B+(1-\lambda) A)+(1-t) A] d t \\
& =\int_{\lambda}^{1} f[u B+(1-u) A] d u+\int_{0}^{\lambda} f[u B+(1-u) A] d u \\
& =\int_{0}^{1} f[u B+(1-u) A] d u
\end{aligned}
$$

and the identity (2.5) is proved.

We have now the following generalization of (1.1):

Theorem 4. Let $f: I \rightarrow \mathbb{R}$ be an operator convex function on the interval $I$. Then for any selfadjoint operators $A$ and $B$ with spectra in $I$ and for any $\lambda \in[0,1]$ we have the inequalities

$$
\begin{aligned}
f\left(\frac{A+B}{2}\right) & \leq(1-\lambda) f\left[\frac{(1-\lambda) A+(1+\lambda) B}{2}\right]+\lambda f\left[\frac{(2-\lambda) A+\lambda B}{2}\right] \\
& \leq \int_{0}^{1} f((1-t) A+t B) d t \\
& \leq \frac{1}{2}[f((1-\lambda) A+\lambda B)+(1-\lambda) f(B)+\lambda f(A)] \\
& \leq \frac{f(A)+f(B)}{2} .
\end{aligned}
$$

Proof. Using the Hermite-Hadamard inequality (1.1) we have

$$
\begin{aligned}
f\left[\frac{(1-\lambda) A+(1+\lambda) B}{2}\right] & \leq \int_{0}^{1} f[(1-t)((1-\lambda) A+\lambda B)+t B] d t \\
& \leq \frac{f((1-\lambda) A+\lambda B)+f(B)}{2}
\end{aligned}
$$

and

$$
\begin{aligned}
f\left[\frac{(2-\lambda) A+\lambda B}{2}\right] & \leq \int_{0}^{1} f[(1-t) A+t((1-\lambda) A+\lambda B)] d t \\
& \leq \frac{f(A)+f((1-\lambda) A+\lambda B)}{2}
\end{aligned}
$$

for any $\lambda \in[0,1]$. 
If we multiply inequality (2.7) by $1-\lambda$ and (2.8) by $\lambda$, add the obtained inequalities and use representation (2.5), then we get

$$
\begin{aligned}
& (1-\lambda) f\left[\frac{(1-\lambda) A+(1+\lambda) B}{2}\right]+\lambda f\left[\frac{(2-\lambda) A+\lambda B}{2}\right] \\
& \leq \int_{0}^{1} f((1-t) A+t B) d t \\
& \leq(1-\lambda) \frac{f((1-\lambda) A+\lambda B)+f(B)}{2}+\lambda \frac{f(A)+f((1-\lambda) A+\lambda B)}{2},
\end{aligned}
$$

which proves the second and third inequalities in (2.6).

By the operator convexity of $f$ we have

$$
\begin{aligned}
& (1-\lambda) f\left[\frac{(1-\lambda) A+(1+\lambda) B}{2}\right]+\lambda f\left[\frac{(2-\lambda) A+\lambda B}{2}\right] \\
& \geq f\left[(1-\lambda) \frac{(1-\lambda) A+(1+\lambda) B}{2}+\lambda \frac{(2-\lambda) A+\lambda B}{2}\right]=f\left(\frac{A+B}{2}\right)
\end{aligned}
$$

and

$$
\begin{aligned}
& \frac{1}{2}[f((1-\lambda) A+\lambda B)+(1-\lambda) f(B)+\lambda f(A)] \\
& \leq \frac{1}{2}[(1-\lambda) f(A)+\lambda f(B)+(1-\lambda) f(B)+\lambda f(A)]=\frac{f(A)+f(B)}{2}
\end{aligned}
$$

that prove the first and last inequality in (2.6).

We have:

Corollary 2. Let $f: I \rightarrow \mathbb{R}$ be an operator convex function on the interval $I$. Then for any selfadjoint operators $A$ and $B$ with spectra in I and $\Phi \in \mathfrak{P}_{N}[\mathcal{B}(H), \mathcal{B}(K)]$ we have

$$
\begin{aligned}
\Phi\left(f\left(\frac{A+B}{2}\right)\right) & \leq(1-\lambda) \Phi\left(f\left[\frac{(1-\lambda) A+(1+\lambda) B}{2}\right]\right)+\lambda \Phi\left(f\left[\frac{(2-\lambda) A+\lambda B}{2}\right]\right) \\
& \leq \int_{0}^{1} \Phi(f((1-t) A+t B)) d t \\
& \leq \frac{1}{2}[\Phi(f((1-\lambda) A+\lambda B))+(1-\lambda) \Phi(f(B))+\lambda \Phi(f(A))] \\
& \leq \frac{\Phi(f(A))+\Phi(f(B))}{2}
\end{aligned}
$$

for any $\lambda \in[0,1]$.

\section{Bounds for HH-Difference}

We consider the difference functional

$$
J_{n}(\mathbf{p} ; \mathbf{A}, f, I):=\sum_{j=1}^{n} p_{j} f\left(A_{j}\right)-P_{n} f\left(\frac{1}{P_{n}} \sum_{j=1}^{n} p_{j} A_{j}\right)
$$

where $\mathbf{p}=\left(p_{1}, \ldots, p_{n}\right), p_{j} \geq 0$ with $j \in\{1, \ldots, n\}$ and $P_{n}>0, \mathbf{A}=\left(A_{1}, \ldots, A_{n}\right)$ is an $n$-tuple of selfadjoint operators with $\operatorname{Sp}\left(A_{j}\right) \subseteq I$ for $j \in\{1, \ldots, n\}$ and $f: I \rightarrow \mathbb{R}$ is a operator convex function defined on the interval $I$.

We denote by $\mathcal{P}_{n}^{+}$the set of all $n$-tuples $\mathbf{p}=\left(p_{1}, \ldots, p_{n}\right), p_{j} \geq 0$ with $j \in\{1, \ldots, n\}$ and $P_{n}>0$. For $\mathbf{p}, \mathbf{q} \in \mathcal{P}_{n}^{+}$ we denote $\mathbf{p} \geq \mathbf{q}$ if $p_{j} \geq q_{j}$ for any $j \in\{1, \ldots, n\}$.

In [7] we established the following properties of the functional $J_{n}(\cdot ; \mathbf{A}, f, I)$ : 
Theorem 5. Assume that $f: I \rightarrow \mathbb{R}$ is an operator convex function and $\mathbf{A}=\left(A_{1}, \ldots, A_{n}\right)$ an $n$-tuple of selfadjoint operators with $\operatorname{Sp}\left(A_{j}\right) \subseteq I$, then for any $\mathbf{p}, \mathbf{q} \in \mathcal{P}_{n}^{+}$we have

$$
J_{n}(\mathbf{p}+\mathbf{q} ; \mathbf{A}, f, I) \geq J_{n}(\mathbf{p} ; \mathbf{A}, f, I)+J_{n}(\mathbf{q} ; \mathbf{A}, f, I) \geq 0,
$$

i.e., $J_{n}(\cdot ; \mathbf{A}, f, I)$ is a super-additive functional in the operator order.

Moreover, if $\mathbf{p}, \mathbf{q} \in \mathcal{P}_{n}^{+}$with $\mathbf{p} \geq \mathbf{q}$, then also

$$
J_{n}(\mathbf{p} ; \mathbf{A}, f, I) \geq J_{n}(\mathbf{q} ; \mathbf{A}, f, I) \geq 0,
$$

i.e., $J_{n}(\cdot ; \mathbf{A}, f, I)$ is a monotonic functional in the operator order.

The following boundedness property also holds:

Corollary 3. Assume that the function $f: I \rightarrow \mathbb{R}$ is operator convex and the n-tuple of selfadjoint operators $\left(A_{1}, \ldots, A_{n}\right)$ satisfies the condition $\operatorname{Sp}\left(A_{j}\right) \subseteq$ I for any $j \in\{1, \ldots, n\}$. If $\mathbf{p}, \mathbf{q} \in \mathcal{P}_{n}^{+}$and there exists the positive constants $m, M$ such that

$$
m \mathbf{q} \leq \mathbf{p} \leq M \mathbf{q},
$$

then

$$
m J_{n}(\mathbf{q} ; \mathbf{A}, f, I) \leq J_{n}(\mathbf{p} ; \mathbf{A}, f, I) \leq M J_{n}(\mathbf{q} ; \mathbf{A}, f, I)
$$

in the operator order.

We observe that if all $q_{j}>0, j \in\{1, \ldots, n\}$, then we have the inequality

$$
\begin{aligned}
\min _{j \in\{1, \ldots, n\}}\left\{\frac{p_{j}}{q_{j}}\right\} J_{n}(\mathbf{q} ; \mathbf{A}, f, I) & \leq J_{n}(\mathbf{p} ; \mathbf{A}, f, I) \\
& \leq \max _{j \in\{1, \ldots, n\}}\left\{\frac{p_{j}}{q_{j}}\right\} J_{n}(\mathbf{q} ; \mathbf{A}, f, I)
\end{aligned}
$$

in the operator order.

In particular, by (3.6) for $n=2, p_{1}=1-p, p_{2}=p, q_{1}=1-q$ and $q_{2}=q$ with $p \in[0,1]$ and $q \in(0,1)$ we get

$$
\begin{aligned}
& \min \left\{\frac{p}{q}, \frac{1-p}{1-q}\right\}[(1-q) f(A)+q f(B)-f((1-q) A+q B)] \\
& \leq[(1-p) f(A)+p f(B)-f((1-p) A+p B)] \\
& \leq \max \left\{\frac{p}{q}, \frac{1-p}{1-q}\right\}[(1-q) f(A)+q f(B)-f((1-q) A+q B)]
\end{aligned}
$$

for any selfadjoint operators $A$ and $B$ with spectra in $I$.

If we take $q=\frac{1}{2}$ in (1.1), then we get

$$
\begin{aligned}
2 \min \{t, 1-t\}\left[\frac{f(A)+f(B)}{2}-f\left(\frac{A+B}{2}\right)\right] & \leq[(1-t) f(A)+t f(B)-f((1-t) A+t B)] \\
& \leq 2 \max \{t, 1-t\}\left[\frac{f(A)+f(B)}{2}-f\left(\frac{A+B}{2}\right)\right]
\end{aligned}
$$

for any selfadjoint operators $A$ and $B$ with spectra in $I$ and $t \in[0,1]$.

If we take in (3.7) the map $\Phi$, then we have

$$
\begin{aligned}
& \min \left\{\frac{p}{q}, \frac{1-p}{1-q}\right\}[(1-q) \Phi(f(A))+q \Phi(f(B))-\Phi(f((1-q) A+q B))] \\
& \leq[(1-p) \Phi(f(A))+p \Phi(f(B))-\Phi(f((1-p) A+p B))] \\
& \leq \max \left\{\frac{p}{q}, \frac{1-p}{1-q}\right\}[(1-q) \Phi(f(A))+q \Phi(f(B))-\Phi(f((1-q) A+q B))]
\end{aligned}
$$


for any $\Phi \in \mathfrak{P}_{N}[\mathcal{B}(H), \mathcal{B}(K)]$.

The following result provides some upper and lower bounds for the $\mathrm{HH}$-difference

$$
\frac{f(A)+f(B)}{2}-\int_{0}^{1} f((1-t) A+t B) d t .
$$

Theorem 6. Let $f: I \rightarrow \mathbb{R}$ be an operator convex function on the interval $I$. Then for any selfadjoint operators $A$ and $B$ with spectra in $I$ we have the inequality

$$
\begin{aligned}
\frac{1}{2}[(1-q) f(A)+q f(B)-f((1-q) A+q B)] & \leq \frac{f(A)+f(B)}{2}-\int_{0}^{1} f((1-t) A+t B) d t \\
& \leq \frac{1}{2} \frac{q^{2}-q+1}{q(1-q)}[(1-q) f(A)+q f(B)-f((1-q) A+q B)]
\end{aligned}
$$

for any $q \in(0,1)$.

Proof. From (3.7) we have

$$
\begin{aligned}
& \min \left\{\frac{t}{q}, \frac{1-t}{1-q}\right\}[(1-q) f(A)+q f(B)-f((1-q) A+q B)] \\
& \leq[(1-t) f(A)+t f(B)-f((1-t) A+t B)] \\
& \leq \max \left\{\frac{t}{q}, \frac{1-t}{1-q}\right\}[(1-q) f(A)+q f(B)-f((1-q) A+q B)]
\end{aligned}
$$

with $t \in[0,1]$ and $q \in(0,1)$.

If we integrate over $t \in[0,1]$ the inequality (3.11), then we get

$$
\begin{aligned}
& {[(1-q) f(A)+q f(B)-f((1-q) A+q B)] \int_{0}^{1} \min \left\{\frac{t}{q}, \frac{1-t}{1-q}\right\} d t} \\
& \leq \frac{f(A)+f(B)}{2}-\int_{0}^{1} f((1-t) A+t B) d t \\
& \leq[(1-q) f(A)+q f(B)-f((1-q) A+q B)] \int_{0}^{1} \max \left\{\frac{t}{q}, \frac{1-t}{1-q}\right\} d t
\end{aligned}
$$

for any $A, B$ with spectra in $I$ and $q \in(0,1)$.

Observe that

$$
\frac{t}{q}-\frac{1-t}{1-q}=\frac{t-q}{q(1-q)}
$$

showing that

and

$$
\min \left\{\frac{t}{q}, \frac{1-t}{1-q}\right\}=\left\{\begin{array}{c}
\frac{t}{q} \text { if } 0 \leq t \leq q \leq 1 \\
\frac{1-t}{1-q} \text { if } 0 \leq q \leq t \leq 1
\end{array}\right.
$$

Then

$$
\max \left\{\frac{t}{q}, \frac{1-t}{1-q}\right\}=\left\{\begin{array}{l}
\frac{1-t}{1-q} \text { if } 0 \leq t \leq q \leq 1 \\
\frac{t}{q} \text { if } 0 \leq q \leq t \leq 1
\end{array}\right.
$$

$$
\begin{aligned}
\int_{0}^{1} \min \left\{\frac{t}{q}, \frac{1-t}{1-q}\right\} d t & =\int_{0}^{q} \frac{t}{q} d t+\int_{q}^{1} \frac{1-t}{1-q} d t \\
& =\frac{q^{2}}{2 q}+\frac{1}{1-q}\left(1-q-\left(\frac{1-q^{2}}{2}\right)\right)=\frac{1}{2}
\end{aligned}
$$


and

$$
\begin{aligned}
\int_{0}^{1} \max \left\{\frac{t}{q}, \frac{1-t}{1-q}\right\} d t & =\int_{0}^{q} \frac{1-t}{1-q} d t+\int_{q}^{1} \frac{t}{q} d t \\
& =\frac{1}{1-q}\left(q-\frac{q^{2}}{2}\right)+\frac{1-q^{2}}{2 q} \\
& =\frac{q^{2}-q+1}{2 q(1-q)}
\end{aligned}
$$

and by (3.12) we obtain the desired result (3.10).

Corollary 4. Let $f: I \rightarrow \mathbb{R}$ be an operator convex function on the interval $I$. Then for any selfadjoint operators $A$ and $B$ with spectra in $I$ and $\Phi \in \mathfrak{P}_{N}[\mathcal{B}(H), \mathcal{B}(K)]$ we have

$$
\begin{aligned}
& \frac{1}{2}[(1-q) \Phi(f(A))+q \Phi(f(B))-\Phi(f((1-q) A+q B))] \\
& \leq \frac{\Phi(f(A))+\Phi(f(B))}{2}-\int_{0}^{1} \Phi(f((1-t) A+t B)) d t \\
& \leq \frac{1}{2} \frac{q^{2}-q+1}{q(1-q)}[(1-q) \Phi(f(A))+q \Phi(f(B))-\Phi(f((1-q) A+q B))] .
\end{aligned}
$$

We also have the following bounds for the other HH-difference

$$
\int_{0}^{1} f((1-t) A+t B) d t-f\left(\frac{A+B}{2}\right) .
$$

Theorem 7. Let $f: I \rightarrow \mathbb{R}$ be an operator convex function on the interval I. Then for any selfadjoint operators $A$ and $B$ with spectra in I we have the inequality

$$
\begin{aligned}
& \frac{1}{2 q(1-q)} \min \{1-q, q\}\left[\int_{0}^{1} f((1-t) A+t B) d t-\frac{1}{1-2 q} \int_{q}^{1-q} f((1-s) A+s B) d s\right] \\
& \leq \int_{0}^{1} f((1-t) A+t B) d t-f\left(\frac{A+B}{2}\right) \\
& \leq \frac{1}{2 q(1-q)} \max \{1-q, q\}\left[\int_{0}^{1} f((1-t) A+t B) d t-\frac{1}{1-2 q} \int_{q}^{1-q} f((1-s) A+s B) d s\right]
\end{aligned}
$$

or, equivalently

$$
\begin{aligned}
& \frac{2 q(1-q)}{\max \{1-q, q\}}\left[\int_{0}^{1} f((1-t) A+t B) d t-f\left(\frac{A+B}{2}\right)\right] \\
& \leq \int_{0}^{1} f((1-t) A+t B) d t-\frac{1}{1-2 q} \int_{q}^{1-q} f((1-s) A+s B) d s \\
& \leq \frac{2 q(1-q)}{\min \{1-q, q\}}\left[\int_{0}^{1} f((1-t) A+t B) d t-f\left(\frac{A+B}{2}\right)\right]
\end{aligned}
$$

for any $q \in(0,1), q \neq \frac{1}{2}$. 
Proof. If we take in (3.7) $p=\frac{1}{2}$, then we have

$$
\begin{aligned}
& \frac{1}{2 q(1-q)} \min \{1-q, q\}[(1-q) f(A)+q f(B)-f((1-q) A+q B)] \\
& \leq \frac{f(A)+f(B)}{2}-f\left(\frac{A+B}{2}\right) \\
& \leq \frac{1}{2 q(1-q)} \max \{1-q, q\}[(1-q) f(A)+q f(B)-f((1-q) A+q B)]
\end{aligned}
$$

for any $A, B$ with spectra in $I$ and $q \in(0,1)$.

If we replace $A$ by $(1-t) A+t B$ and $B$ by $t A+(1-t) B$ in (3.16), then we get

$$
\begin{aligned}
& \frac{1}{2 q(1-q)} \min \{1-q, q\}[(1-q) f((1-t) A+t B)+q f(t A+(1-t) B) \\
& -f((1-q)[(1-t) A+B t]+q[t A+(1-t) B])] \\
& \leq \frac{f((1-t) A+t B)+f(t A+(1-t) B)}{2}-f\left(\frac{A+B}{2}\right) \\
& \leq \frac{1}{2 q(1-q)} \max \{1-q, q\}[(1-q) f((1-t) A+t B)+q f(t A+(1-t) B) \\
& -f((1-q)[(1-t) A+B t]+q[t A+(1-t) B])]
\end{aligned}
$$

for any $A, B \in C, t \in[0,1]$ and $q \in(0,1)$.

If we take the integral over $t \in[0,1]$ in (3.17) and take into account that

$$
\int_{0}^{1} f((1-t) A+t B) d t=\int_{0}^{1} f(t A+(1-t) B) d t
$$

we get

$$
\begin{aligned}
& \frac{1}{2 q(1-q)} \min \{1-q, q\}\left[\int_{0}^{1} f((1-t) A+t B) d t-\int_{0}^{1} f((1-q)[(1-t) A+t B]+q[t A+(1-t) B]) d t\right] \\
& \leq \int_{0}^{1} f((1-t) A+t B) d t-f\left(\frac{A+B}{2}\right) \\
& \leq \frac{1}{2 q(1-q)} \max \{1-q, q\}\left[\int_{0}^{1} f((1-t) A+t B) d t-\int_{0}^{1} f((1-q)[(1-t) A+t B]+q[t A+(1-t) B]) d t\right]
\end{aligned}
$$

or any $A, B$ with spectra in $I$ and $q \in(0,1)$.

Observe that for any $A, B$ with spectra in $I, t \in[0,1]$ and $q \in(0,1)$ we have

$$
(1-q)[(1-t) A+t B]+q[t A+(1-t) B]=[(1-q)(1-t)+q t] A+[(1-q) t+(1-t) q] B
$$

and by putting $s:=(1-q) t+(1-t) q$, for $q \neq \frac{1}{2}$ we have

$$
[(1-q)(1-t)+q t] A+[(1-q) t+(1-t) q] B=(1-s) A+s B .
$$

If $q \neq \frac{1}{2}$, then $s$ is a change of variable, $d s=(1-2 q) d t$ and we have for any $A, B$ with spectra in $I$ that

$$
\int_{0}^{1} f((1-q)[(1-t) A+t B]+q[t A+(1-t) B]) d t=\frac{1}{1-2 q} \int_{q}^{1-q} f((1-s) A+s B) d s .
$$

On making use of (3.18) we get the desired result (3.14). 
Corollary 5. Let $f: I \rightarrow \mathbb{R}$ be an operator convex function on the interval $I$. Then for any selfadjoint operators $A$ and $B$ with spectra in $I$ and $\Phi \in \mathfrak{P}_{N}[\mathcal{B}(H), \mathcal{B}(K)]$ we have

$$
\begin{aligned}
& \frac{2 q(1-q)}{\max \{1-q, q\}}\left[\int_{0}^{1} \Phi(f((1-t) A+t B)) d t-\Phi\left(f\left(\frac{A+B}{2}\right)\right)\right] \\
& \leq \int_{0}^{1} \Phi(f((1-t) A+t B)) d t-\frac{1}{1-2 q} \int_{q}^{1-q} \Phi(f((1-s) A+s B)) d s \\
& \leq \frac{2 q(1-q)}{\min \{1-q, q\}}\left[\int_{0}^{1} \Phi(f((1-t) A+t B)) d t-\Phi\left(f\left(\frac{A+B}{2}\right)\right)\right]
\end{aligned}
$$

for any $q \in(0,1), q \neq \frac{1}{2}$.

Remark 1. If we take $q=\frac{1}{4}$ in (3.15) and (3.19), then we get

$$
\begin{aligned}
\frac{1}{2}\left[\int_{0}^{1} f((1-t) A+t B) d t-f\left(\frac{A+B}{2}\right)\right] & \leq \int_{0}^{1} f((1-t) A+t B) d t-2 \int_{1 / 4}^{3 / 4} f((1-s) A+s B) d s \\
& \leq \frac{3}{2}\left[\int_{0}^{1} f((1-t) A+t B) d t-f\left(\frac{A+B}{2}\right)\right]
\end{aligned}
$$

and

$$
\frac{1}{2}\left[\int_{0}^{1} \Phi(f((1-t) A+t B)) d t-\Phi\left(f\left(\frac{A+B}{2}\right)\right)\right] \leq \int_{0}^{1} \Phi(f((1-t) A+t B)) d t-2 \int_{1 / 4}^{3 / 4} \Phi(f((1-s) A+s B)) d s
$$

$$
\leq \frac{3}{2}\left[\int_{0}^{1} \Phi(f((1-t) A+t B)) d t-\Phi\left(f\left(\frac{A+B}{2}\right)\right)\right]
$$

for any $A, B$ with spectra in $I$ and $\Phi \in \mathfrak{P}_{N}[\mathcal{B}(H), \mathcal{B}(K)]$.

\section{Some Examples}

The function $f(t)=t^{r}$ is operator convex on $(0, \infty)$ if either $1 \leq r \leq 2$ or $-1 \leq r \leq 0$ and is operator concave on $(0, \infty)$ if $0 \leq r \leq 1$.

If we write the inequality (2.3) for the power $1 \leq r \leq 2$ (or $-1 \leq r \leq 0$ ) we have

$$
\int_{0}^{1}((1-t) \Phi(A)+t \Phi(B))^{r} d t \leq \int_{0}^{1} \Phi\left(((1-t) A+t B)^{r}\right) d t
$$

where $\Phi \in \mathfrak{P}_{N}[\mathcal{B}(H), \mathcal{B}(K)]$ and $A, B \in \mathcal{B}^{+}(H)\left(A, B \in \mathcal{B}^{++}(H)\right)$. In the case $0 \leq r \leq 1$ the inequalities reverse in (4.1). 
If we write the inequality (2.9) for the power $1 \leq r \leq 2$ (or $-1 \leq r \leq 0$ ) we have

$$
\begin{aligned}
\Phi\left(\left(\frac{A+B}{2}\right)^{r}\right) & \leq(1-\lambda) \Phi\left(\left[\frac{(1-\lambda) A+(1+\lambda) B}{2}\right]^{r}\right)+\lambda \Phi\left(\left[\frac{(2-\lambda) A+\lambda B}{2}\right]^{r}\right) \\
& \leq \int_{0}^{1} \Phi\left(((1-t) A+t B)^{r}\right) d t \\
& \leq \frac{1}{2}\left[\Phi\left(((1-\lambda) A+\lambda B)^{r}\right)+(1-\lambda) \Phi\left(B^{r}\right)+\lambda \Phi\left(A^{r}\right)\right] \\
& \leq \frac{\Phi\left(A^{r}\right)+\Phi\left(B^{r}\right)}{2},
\end{aligned}
$$

where $\lambda \in[0,1], \Phi \in \mathfrak{P}_{N}[\mathcal{B}(H), \mathcal{B}(K)]$ and $A, B \in \mathcal{B}^{+}(H)\left(A, B \in \mathcal{B}^{++}(H)\right)$. In the case $0 \leq r \leq 1$ the inequalities reverse in (4.2).

If we write the inequality (3.9) for the power $1 \leq r \leq 2$ (or $-1 \leq r \leq 0$ ) we get for $p \in[0,1], q \in(0,1)$ that

$$
\begin{aligned}
& \min \left\{\frac{p}{q}, \frac{1-p}{1-q}\right\}\left[(1-q) \Phi\left(A^{r}\right)+q \Phi\left(B^{r}\right)-\Phi\left(((1-q) A+q B)^{r}\right)\right] \\
& \leq\left[(1-p) \Phi\left(A^{r}\right)+p \Phi\left(B^{r}\right)-\Phi\left(((1-p) A+p B)^{r}\right)\right] \\
& \leq \max \left\{\frac{p}{q}, \frac{1-p}{1-q}\right\}\left[(1-q) \Phi\left(A^{r}\right)+q \Phi\left(B^{r}\right)-\Phi\left(((1-q) A+q B)^{r}\right)\right]
\end{aligned}
$$

where $\Phi \in \mathfrak{P}_{N}[\mathcal{B}(H), \mathcal{B}(K)]$ and $A, B \in \mathcal{B}^{+}(H)\left(A, B \in \mathcal{B}^{++}(H)\right)$.

From (3.13) we have for $1 \leq r \leq 2$ (or $-1 \leq r \leq 0$ ) that

$$
\begin{aligned}
& \frac{1}{2}\left[(1-q) \Phi\left(A^{r}\right)+q \Phi\left(B^{r}\right)-\Phi\left(((1-q) A+q B)^{r}\right)\right] \\
& \leq \frac{\Phi\left(A^{r}\right)+\Phi\left(B^{r}\right)}{2}-\int_{0}^{1} \Phi\left(((1-t) A+t B)^{r}\right) d t \\
& \leq \frac{1}{2} \frac{q^{2}-q+1}{q(1-q)}\left[(1-q) \Phi\left(A^{r}\right)+q \Phi\left(B^{r}\right)-\Phi\left(((1-q) A+q B)^{r}\right)\right]
\end{aligned}
$$

while from(3.19) we have that

$$
\begin{aligned}
& \frac{2 q(1-q)}{\max \{1-q, q\}}\left[\int_{0}^{1} \Phi\left(((1-t) A+t B)^{r}\right) d t-\Phi\left(\left(\frac{A+B}{2}\right)^{r}\right)\right] \\
& \leq \int_{0}^{1} \Phi\left(((1-t) A+t B)^{r}\right) d t-\frac{1}{1-2 q} \int_{q}^{1-q} \Phi\left(((1-s) A+s B)^{r}\right) d s \\
& \leq \frac{2 q(1-q)}{\min \{1-q, q\}}\left[\int_{0}^{1} \Phi\left(((1-t) A+t B)^{r}\right) d t-\Phi\left(\left(\frac{A+B}{2}\right)^{r}\right)\right],
\end{aligned}
$$

where $p \in[0,1], q \in(0,1), \Phi \in \mathfrak{P}_{N}[\mathcal{B}(H), \mathcal{B}(K)]$ and $A, B \in \mathcal{B}^{+}(H)\left(A, B \in \mathcal{B}^{++}(H)\right)$.

The function $f(t)=-\ln t$ is operator convex on $(0, \infty)$. Then by $(2.3)$ we have

$$
\int_{0}^{1} \ln ((1-t) \Phi(A)+t \Phi(B)) d t \geq \int_{0}^{1} \Phi(\ln ((1-t) A+t B)) d t
$$


while by (2.9) we have, for $\lambda \in[0,1]$ that

$$
\begin{aligned}
\Phi\left(\ln \left(\frac{A+B}{2}\right)\right) & \geq(1-\lambda) \Phi\left(\ln \left[\frac{(1-\lambda) A+(1+\lambda) B}{2}\right]\right)+\lambda \Phi\left(\ln \left[\frac{(2-\lambda) A+\lambda B}{2}\right]\right) \\
& \geq \int_{0}^{1} \Phi(\ln ((1-t) A+t B)) d t \\
& \geq \frac{1}{2}[\Phi(\ln ((1-\lambda) A+\lambda B))+(1-\lambda) \Phi(\ln (B))+\lambda \Phi(\ln (A))] \\
& \geq \frac{\Phi(\ln (A))+\Phi(\ln (B))}{2}
\end{aligned}
$$

where $\Phi \in \mathfrak{P}_{N}[\mathcal{B}(H), \mathcal{B}(K)]$ and $A, B \in \mathcal{B}^{++}(H)$.

From (3.9) we have for $p \in[0,1], q \in(0,1)$ that

$$
\begin{aligned}
& \min \left\{\frac{p}{q}, \frac{1-p}{1-q}\right\}[\Phi(\ln ((1-q) A+q B))-(1-q) \Phi(\ln (A))-q \Phi(\ln (B))] \\
& \leq[\Phi(\ln ((1-p) A+p B))-(1-p) \Phi(\ln (A))-p \Phi(\ln (B))] \\
& \leq \max \left\{\frac{p}{q}, \frac{1-p}{1-q}\right\}[\Phi(\ln ((1-q) A+q B))-(1-q) \Phi(\ln (A))-q \Phi(\ln (B))],
\end{aligned}
$$

from (3.13) we have

$$
\begin{aligned}
& \frac{1}{2}[\Phi(\ln ((1-q) A+q B))-(1-q) \Phi(\ln (A))-q \Phi(\ln (B))] \\
& \leq \int_{0}^{1} \Phi(\ln ((1-t) A+t B)) d t-\frac{\Phi(\ln (A))+\Phi(\ln (B))}{2} \\
& \leq \frac{1}{2} \frac{q^{2}-q+1}{q(1-q)}[\Phi(\ln ((1-q) A+q B))-(1-q) \Phi(\ln (A))-q \Phi(\ln (B))] .
\end{aligned}
$$

while from (3.19)

$$
\begin{aligned}
& \frac{2 q(1-q)}{\max \{1-q, q\}}\left[\Phi\left(\ln \left(\frac{A+B}{2}\right)\right)-\int_{0}^{1} \Phi(\ln ((1-t) A+t B)) d t\right] \\
& \leq \frac{1}{1-2 q} \int_{q}^{1-q} \Phi(\ln ((1-s) A+s B)) d s-\int_{0}^{1} \Phi(\ln ((1-t) A+t B)) d t \\
& \leq \frac{2 q(1-q)}{\min \{1-q, q\}}\left[\Phi\left(\ln \left(\frac{A+B}{2}\right)\right)-\int_{0}^{1} \Phi(\ln ((1-t) A+t B)) d t\right]
\end{aligned}
$$

where $\Phi \in \mathfrak{P}_{N}[\mathcal{B}(H), \mathcal{B}(K)]$ and $A, B \in \mathcal{B}^{++}(H)$.

\section{References}

[1] V. Bacak and R. Türkmen, Refinements of Hermite-Hadamard type inequalities for operator convex functions. J. Inequal. Appl. 2013, 2013:262, 10 pp.

[2] B. Li, Refinements of Hermite-Hadamard's type inequalities for operator convex functions. Int. J. Contemp. Math. Sci. 8 (2013), no. 9-12, 463-467.

[3] M. D. Choi, Positive linear maps on C*-algebras. Canad. J. Math. 24 (1972), 520-529.

[4] S. S. Dragomir, Hermite-Hadamard's type inequalities for operator convex functions, Applied Mathematics and Computation, 218 (2011), Issue 3, pp. 766-772. 
[5] S. S. Dragomir, Operator Inequalities of the Jensen, Čebyšev and Grüss Type. Springer Briefs in Mathematics. Springer, New York, 2012. xii+121 pp. ISBN: 978-1-4614-1520-6.

[6] S. S. Dragomir, Some inequalities of Hermite-Hadamard type for convex functions of commuting selfadjoint operators in Hilbert spaces. Boll. Unione Mat. Ital. (9) 6 (2013), no. 3, 491-511.

[7] S. S. Dragomir, Some inequalities of Jensen type for operator convex functions in Hilbert spaces, Advances in Inequalities and Applications, Vol 2, No 1 (2013), 105-123. [Online http://scik.org/index.php/aia/article/view/904] Preprint RGMIA Res. Rep. Coll. 15 (2012), Art. 40, [Online http://rgmia.org/papers/v15/v15a40.pdf].

[8] A. G. Ghazanfari, The Hermite-Hadamard type inequalities for operator s-convex functions. J. Adv. Res. Pure Math. 6 (2014), no. 3, 52-61

[9] A. G. Ghazanfari and A. Barani, Some Hermite-Hadamard type inequalities for the product of two operator preinvex functions. Banach J. Math. Anal. 9 (2015), no. 2, 9-20.

[10] M. S. Moslehian, Matrix Hermite-Hadamard type inequalities, Houston J. Math 39 (2013) no 1, 178-189.

[11] P. Skoufranis, Completely Positive Maps, [Online http://www.math.tamu.edu/ pskoufra/ OANotesCompletelyPositiveMaps.pdf].

[12] J. Pečarić, T. Furuta, J. Mićić Hot and Y. Seo, Mond-Pečarić Method in Operator Inequalities. Inequalities for Bounded Selfadjoint Operators on a Hilbert Space, Element, Zagreb, 2005.

[13] S.-H. Wang and X.-M. Liu, Hermite-Hadamard type inequalities for operator s-preinvex functions. J. Nonlinear Sci. Appl. 8 (2015), no. 6, 1070-1081. 